FORMATION Formation emploi

Revue française de sciences sociales

143 | Juillet-Septembre 2018

Le retour en formation : une vraie chance?

\title{
Accompagner les « jeunes en errance » ou adapter les normes de l'insertion
}

Young homeless people and social accompaniment in France : how to deal with youth professional integration norms?

"Instabile"Jugendliche begleiten oder Eingliederungsstandards anpassen

Acompañar a los "jóvenes errantes" o adaptar las normas de inserción

\section{Céline Rothé}

\section{OpenEdition}

Journals

Édition électronique

URL : http://journals.openedition.org/formationemploi/6302

DOI : 10.4000/formationemploi.6302

ISSN : 2107-0946

Éditeur

La Documentation française

Édition imprimée

Date de publication : 20 novembre 2018

Pagination : 161-182

ISSN : 0759-6340

Référence électronique

Céline Rothé, « Accompagner les « jeunes en errance » ou adapter les normes de l'insertion »,

Formation emploi [En ligne], 143 | Juillet-Septembre 2018, mis en ligne le 20 novembre 2020, consulté le 06 novembre 2020. URL : http://journals.openedition.org/formationemploi/6302 ; DOI : https:// doi.org/10.4000/formationemploi.6302

(c) Tous droits réservés 


\title{
Accompagner les « jeunes en errance » ou adapter les normes de l'insertion
}

\author{
CÉLINE Rothé \\ Université de Rennes, École des Hautes Études en Santé Publique, CNRS, Arènes Unité Mixte \\ de Recherche 6051, F-35000 Rennes, France
}

Résumé

Accompagner les « jeunes en errance » ou adapter les normes de l'insertion

En France, la politique d'accompagnement à la formation et à l'emploi est essentiellement formalisée autour des compétences à acquérir pour s’insérer sur le marché de l'emploi. Elle met ainsi à l'écart les jeunes qui ne peuvent ou ne veulent pas recourir aux dispositifs d'aide à l'insertion. Au travers de l'analyse du fonctionnement des structures dites "à bas seuil d'exigence ", nous verrons comment une politique d'aide à l'insertion des jeunes les plus marginalisés est malgré tout mise en œuvre dans le secteur de l'aide sociale d'urgence.

Mots clés : action sociale, mesure jeune, accompagnement en formation, accompagnement professionnel, condition de vie, insertion sociale, exclusion, jeune en difficulté

Abstract

Young homeless people and social accompaniment in France: how to deal with youth professional integration norms?

French policies for young people professional integration aim to develop specific skills in order to become more employable. This movement excludes young homeless people because they don't endorse professional integration norms. Through the analysis of the social accompaniment in emergency aid, we can show how a concrete integration policy is implemented despite the distance young homeless people nurture toward integration norms.

Keywords: help support, youth employment scheme, coaching in training, coaching, living condition, social integration; exclusion, young person in difficulty

Journal of Economic Literature: I 32 ; I38

Traduction : Auteure 


\section{Introduction}

En France, la situation des jeunes à l'égard de l'insertion sociale se structure largement autour de l'accès à l'autonomie. Celle-ci s'entend comme la capacité à vivre sans l'aide des parents ou de l'État. Avec l'apparition du chômage de masse, en 1975, et les préoccupations grandissantes autour des jeunes en difficulté, depuis les années 1980, les choix politiques se structurent autour de l'accès à l'emploi via la formation. L'emploi, en tant que pourvoyeur de ressources économiques stables, est perçu comme le principal vecteur d'autonomisation pour les jeunes.

Ainsi, ceux qui s’en éloignent, car ils ne rassemblent pas les qualités nécessaires, préoccupent les pouvoirs publics. S'ils ne parviennent pas à compléter leur formation, ils diminuent leurs chances d'accéder à l'autonomie et devront être aidés par l'État ou par leurs proches. Les évolutions actuelles du marché de l'emploi rendent les conditions de leur insertion de plus en plus défavorables, et clivées par le diplôme.

Afin d'aider les jeunes sans qualification à se qualifier, des dispositifs de "seconde chance " apparaissent. Ils agissent dans une logique préventive et curative au sein du parcours scolaire, dans le cadre de la politique de lutte contre le décrochage. Ils proposent des modalités de formation moins exigeantes à certains égards (comme le volume d'heures de cours, les modalités d'enseignement, le nombre d'élèves en classe). En effet, leur objet est aussi de proposer un accompagnement social et éducatif qui leur apprendra à adopter les bons comportements pour être un élève, puis un travailleur (Denecheau \& al., 2015).

D'autres s'adressent aux jeunes sortis sans diplôme de leur scolarité. Il s'agit de les sensibiliser aux exigences du marché de l'emploi, en leur permettant d'acquérir les compétences nécessaires (en termes d'adaptation des comportements et de formation professionnelle) pour une insertion plus aisée. La Garantie jeunes, par exemple, lorsqu'elle est annoncée par le gouvernement en 2013, se propose de sécuriser les parcours d'insertion professionnelle des jeunes vulnérables. On parle alors de NEET (Neither in Employement nor in Education or Training, ou jeunes ni en emploi, ni en études, ni en formation), et on s'adresse aux "jeunes les plus éloignés de l'emploi, en situation de grande précarité".

Toutefois, si ces dispositifs de seconde chance ciblent les jeunes en difficulté, une part d'entre eux leur échappe, trop éloignée de l'école et tenant à distance les attentes normatives liées au statut d'adulte que sont notamment la formation et l'emploi. Les attendus des dispositifs de seconde chance, s'ils sont assouplis au regard des dispositifs classiques de qualification, sont pourtant encore trop exigeants pour une part des jeunes.

En effet, les plus marginalisés d'entre eux ne trouvent pas de sens dans l'élaboration d'un projet d'avenir organisé autour de la formation et de l'emploi et mu par un objectif professionnel. La mise au travail de soi autour de ce projet constitue, pour 
eux, une étape infranchissable qui les amène à éviter les dispositifs de seconde chance et ainsi à échapper à ce pan de l'action publique.

Pourtant, sans être imaginés comme une seconde chance dans l'accès à la qualification et à l'emploi, des dispositifs pensés pour aider dans l'urgence les populations les plus vulnérables se construisent - au travers des pratiques professionnelles de terrain comme des dispositifs d'accompagnement des jeunes les plus marginalisés. L'aide qu'y proposent les professionnels est motivée par le maintien du lien social. Ce lien va ouvrir la possibilité d'un accompagnement, défini en creux de la façon dont il est habituellement pensé pour les jeunes. En effet, l'accès à l'autonomie résidentielle ou économique, la capacité à élaborer du projet et la socialisation aux exigences du monde du travail ne figurent pas dans les objectifs de la relation d'aide. Celle-ci se construit essentiellement autour de la qualité du lien qui s'établit au moment de l'accueil, qui se veut inconditionnel et sans contrepartie (Céfäi \& Gardella, 2011).

Parmi les jeunes qui fréquentent les accueils d'urgence, on retrouve ceux qui ont été nommés "jeunes en errance" par l'action publique (Pattegay, 2001 ; Rothé 2016). Nous les nommerons jeunes marginaux ou zonards, car ils ont en fait connu une forme de socialisation marginalisée ${ }^{1}$ (Parazelli, 2002), dont les normes et les valeurs se sont construites en interaction avec le monde de la rue plus qu'avec les instances traditionnelles de socialisation que sont la famille et l'école.

Des lors, la norme de l'insertion conventionnelle ne semble pas faire sens pour eux (Muniglia et Rothé, 2012). Ces jeunes qui font l'expérience de la "marginalité revendiquée " se tiennent à distance de la norme de l'insertion par la formation et l'emploi et revendiquent le droit à une vie à la marge, tenant à distance les injonctions à l'insertion par l'emploi et le logement (Muniglia, 2015). Socialisés par le contact avec la "zone $»^{2}$, leur univers de valeurs et de références valorise un mode de vie alternatif, fait de débrouille et de défiance à l'égard des normes de l'insertion dans la vie active. De ce fait, ils adhèrent peu aux dispositifs d'accompagnement vers la formation et l'emploi quand ils leur sont proposés en première instance par les travailleurs sociaux. Ils vont davantage se tourner vers des formes d'aide et d'accompagnement moins formelles et moins engageantes, comme c'est le cas dans le secteur de l'urgence sociale.

1. À savoir l'ensemble des pratiques qui constituent un effort individuel d'insertion par la marge (Parazelli, 2002).

2. Tristana Pimor différencie les appellations des jeunes de la rue et rejette notamment celle de "jeunes en errance ", au profit de l'appellation "zonards". Elle définit ainsi la Zone à la fois comme un groupe, comme un espace géographique et comme un ensemble de comportements. "Etre zonard, cétait donc faire partie de la 'Zone.' C'était être autre. C'était être afflieé à ce groupe social qu'ils appelaient 'La Zone' [...]. La Zone, c'était aussi l'espace de mendicité et de rencontre que les zonards occupaient dans la ville; c'était aussi et peut-être surtout pour les acteurs de liberté, une attitude : zoner, c'est-à-dire traîner, buller, prendre son temps. »(Pimor, 2014, p. 18) 
Ces jeunes marginaux vivent le plus souvent "à la rue " et fréquentent massivement le circuit de l'urgence sociale. Depuis leur enfance, ils cumulent une forte précarité économique et une grande fragilité des supports relationnels. Sur le plan familial, ils sont en général issus de milieux très défavorisés et ont, pour beaucoup, vécu des événements traumatisants durant leur enfance, ayant engendré des troubles importants de la relation sociale, un sentiment d'abandon ou de rejet. Une très grande majorité d'entre eux a été prise en charge, à un moment donné, par l'Aide sociale à l'enfance (ASE). À l'adolescence, ils ont expérimenté des situations de rupture avec leur famille ou avec les structures de protection de l'enfance, et se sont retrouvés durablement sans hébergement et sans ressources. Dans la majorité des cas, ils ont un faible niveau d'étude et ont vécu une situation d'échec scolaire (Muniglia \& Rothé, 2013 ; Rothé, 2016). Ainsi, le parcours de ces jeunes est marqué par des ruptures cumulatives et renvoie au processus de "l'apprentissage raté », décrit par Serge Paugam (2008).

\section{Encadré 1. Méthode}

Cet article est issu d'une recherche menée entre 2005 et 2011, dans des structures de l'urgence sociale (accueil de jour, centre d'hébergement, mais aussi équipes mobiles de psychiatrie-précarité et des éducateurs de rue) à destination des personnes vivant dans la rue. Nous nous sommes intéressés aux jeunes usagers de ces dispositifs $\left.{ }^{*}\right)$. Ce travail analyse la relation d'aide qui s'établit entre les jeunes, nommés « jeunes en errance » par les pouvoirs publics, les milieux professionnels dans les années 2000, et les professionnels travaillant dans ces dispositifs. Cependant, dans cet article, nous avons fait le choix de nous concentrer sur les pratiques professionnelles et la façon dont elles font évoluer les cadres de l'action publique sur le terrain. Une trentaine d'entretiens a été menée avec des jeunes dans les accueils de jour et dans la rue, sous forme de récits de vie. Ces entretiens nous ont permis de mettre en évidence le rôle de l'aide d'urgence comme élément de socialisation marginale ${ }^{* *}$. Une soixantaine de professionnels recevant régulièrement ces jeunes, des secteurs de l'insertion sociale et professionnelle et de l'urgence sociale $\left(^{* * *}\right)$, ont également été rencontrés pour des entretiens semi-directifs traitant de leurs pratiques professionnelles dans un contexte de tensions croissantes dans le travail social, mais également de la relation d'aide entretenue avec les jeunes zonards. Ces entretiens seront mobilisés dans l'article, qui traite de l'ajustement des pratiques professionnelles sur le terrain et des effets induits en termes d'accompagnement social. En effet, l'analyse des discours professionnels sur les pratiques nous a permis de souligner le bricolage pragmatique et éthique de l'intervention sociale en direction des jeunes marginalisés

$\left(^{*}\right)$ : Notre terrain nous a permis de traiter de la signification de l'insertion pour les jeunes marginaux eux-mêmes, et notamment de mettre en évidence leur défiance à l'égard d'objectifs comme l'emploi ou le logement. Plus largement, l'analyse de leurs représentations souligne le paradoxe de la plupart des aides proposées : accompagner les jeunes vers des éléments de socialisation que leur marginalité érige en repoussoir (Rothé, op. cit. ; Muniglia, op. cit.).

$\left(^{* *}\right)$ : Pour une analyse plus approfondie de la construction du problème public " jeunes en errance ", voir l'ouvrage Jeunes en errance. Relation d'aide et carrières de marginalité (Rothé, Ibid.).

$\left.{ }^{* * *}\right)$ : Conseillers en mission locale, éducateurs spécialisés, infirmiers, psychologues, psychiatres, agents d'accueil, assistants de service social, conseillers en économie sociale et familiale. 
Nos travaux montrent que ce sont en fait plus les dispositifs de l'urgence sociale, dits " à bas seuil d'exigence ", que les mesures d'insertion ou de "seconde chance " qui parviennent à accompagner durablement ce public socialisé à la vie de la rue (Rothé, 2016). Dans cet article, nous expliquerons pourquoi ces dispositifs deviennent des acteurs incontournables de l'accompagnement de ces jeunes, alors que ce n'est a priori pas une mission de l'urgence sociale. Nous explorerons également la place de l'insertion conventionnelle, via la formation et l'emploi, dans les pratiques professionnelles, entre mise à distance des impératifs normatifs et difficulté à mettre de côté l'insertion professionnelle comme objectif à long terme pour les plus jeunes des usagers.

\section{La structure des aides publiques fragilise les plus vulnérables}

Centrée sur le diplôme et la formation professionnelle, assise sur la famille comme principal support pour les jeunes, l'institutionnalisation de l'insertion rejette vers l'infra-assistance ceux qui ne peuvent activer ces ressources ${ }^{3}$. En France, les politiques de l'insertion se structurent à l'aune des objectifs de la protection sociale juvénile, visant à sécuriser les parcours d'accès à la formation et à l'emploi dans une logique de responsabilisation et d'activation des capacités juvéniles à se mettre au travail (Lima, 2012).

L'assistance n' étant pas accessible en deçà de 25 ans, ceux qui ne seront pas en mesure de mobiliser les ressources nécessaires à une bonne insertion sont, dans ce contexte, particulièrement exclus de toute forme de protection publique. Ils échappent alors au filet de la protection sociale et une trappe s'ouvre, les précipitant dans le secteur de la lutte contre l'exclusion et de l'urgence sociale.

Ces choix publics en matière d'aide aux jeunes expliquent en partie l'absence des jeunes marginalisés dans les dispositifs de seconde chance. Ils n'adhèrent pas - ou ne sont pas en mesure de mobiliser les compétences nécessaires - aux propositions d'accompagnement à la formation et à l'emploi. La mise au travail de soi normée autour de l'idée du projet professionnel ou même du projet d'avenir, l'idée de se mobiliser autour de l'acquisition des compétences nécessaires à l'insertion dans l'emploi vont à l'encontre de leur mode de vie et aussi de leur système normatif qui met notamment à l'écart le travail comme grand organisateur des trajectoires de vie. Dès lors, l'urgence sociale, et

\footnotetext{
3. L'infra-assistance est constituée des aides qui ne sont liées à aucune des formes de protections sociales prodiguées par l'État-providence. Ceux qui en bénéficient ne disposent ni de revenus liés à l'emploi, ni d'aide régulière de l'assistance (en tant quallocataires ou en tant qu'ayants droit). Ils sont l'objet d'une infraintervention, développée par les professionnels des services de la veille et de l'urgence sociale (mais aussi par les associations caritatives, non enquêtées ici), qui leur procure une infra-assistance, c'est-à-dire une aide ponctuelle et de faible importance, en nature ou en argent. Ce type d'intervention prend le relais de l'assistance pour ceux qui n'en relèvent pas faute de pouvoir répondre à ses critères et exigences (Paugam, 2002a).
} 
son accueil sans contrepartie de projet, offre une réponse plus adaptée à leur demande d'aide, qui s'élabore au jour le jour et non pas sur la base d'un projet d'avenir.

\subsection{Une sécurisation des parcours juvéniles assise sur la famille et sur l'emploi.}

Le contexte français de prise en charge de la jeunesse renvoie une partie de la gestion du risque d'insertion vers les familles (Lima, 2004). En effet, les aides financières en direction des jeunes sont largement reversées aux familles, pour qu'elles aident ensuite leurs enfants. Ce fonctionnement laisse peu de place à la sécurisation des parcours de ceux qui ne peuvent bénéficier du soutien familial et qui ne peuvent alors prétendre qu’à des aides financières ponctuelles, conditionnées à un engagement actif dans une démarche d'insertion. Selon cette logique, les moins de vingt-cinq ans ne relèvent pas de l'aide sociale en tant qu'individus citoyens et ne bénéficient donc pas du droit à la subsistance à titre personnel. Par ailleurs, en France, le débat sur la protection sociale des jeunes se structure principalement dans les années 1980, autour d'une logique de Workfare, c'est-à-dire de contrepartie à l'aide sociale sous la forme du travail. Dès lors, le droit à l'assistance, pour les jeunes, trouve sa contrepartie dans l'obligation d'une responsabilisation dans l'accès au travail. De même, la construction française de l'âge de l'insertion se structure autour de deux pôles que sont la formation et l'emploi. La question cruciale de l'insertion des jeunes est donc celle de l'obtention d'un emploi qui conditionne l'accès à l'ensemble de la vie sociale, l'insertion professionnelle étant, en France, un vecteur fort d'intégration. Laccès à un emploi stable constituerait le terme même du processus d'insertion et permettrait d'accéder à un statut social de citoyen responsable et autonome.

Par conséquent, la lutte contre le risque d'insertion, c'est-à-dire de ne pas parvenir à s'insérer par l'activité économique, se mène sur ces deux fronts au travers de la lutte contre la désincitation à la formation, jusque dans les années 1980, puis davantage sur le volet de la lutte contre la désincitation au travail, ensuite (Lima, 2008). Ainsi, la logique du Workfare prévaut dans les décisions publiques qui concernent l'insertion des jeunes. Elle semble ignorer la frange de ceux que les parcours de vie ont durablement tenus éloignés de l'emploi.

La possibilité qu'une part des jeunes ne s'insère pas par l'emploi constitue, à ce moment, un impensé de l'action publique, lié à la représentation de la jeunesse comme une période d'investissement dans l'avenir, suspendue à la qualité de l'insertion professionnelle qui s'y joue.

De fait, le modèle français semble être marqué par une forte valorisation de la formation initiale et induire des trajectoires dominées par l'enjeu du diplôme et du premier emploi. Ce modèle légitime un maintien prolongé dans la dépendance familiale. Il clive les destins sociaux de façon précoce, en laissant son empreinte sur les parcours de jeunesse (Van de Velde, 2008). Les travaux sur les dispositifs de seconde chance ont eux aussi montré l'inci- 
dence du soutien familial sur les « carrières » de jeunes qui en bénéficient, et notamment la variabilité de cette incidence selon les modalités du support familial, l'origine sociale de la famille et son rapport à l'école (Denecheau \& al, op. cit.). L'étude des dispositifs de l'urgence sociale dits "à bas-seuil d'exigence ", largement fréquentés par les jeunes marginaux, révèle les impensés des dispositifs de deuxième chance, visant pourtant à abaisser les exigences de la contractualisation et du projet pour les jeunes les plus vulnérables.

Si la temporalité est allongée et l'accompagnement humain renforcé, ces dimensions n'apparaissent pas adaptées aux jeunes qui cumulent des difficultés sociales, familiales et psychologiques durables. La durée d'accompagnement est trop courte et la visée d'autonomie (donner les compétences pour que la jeune personne puisse engager seule ses démarches) est vécue comme une rupture de lien par des jeunes attachés au lien que noue la relation d'aide plus qu'à l'aide elle-même ${ }^{4}$.

\subsection{Le spectre de l'assistance}

Confronté à une polarisation de la jeunesse ${ }^{5}$, le programme de lutte contre les exclusions, engagé en France en 1998, renforce pourtant l'exclusion des jeunes par le refus d'accorder le RMI (Revenu minimum d'insertion) aux moins de 25 ans (Paugam, 2002b). Cette position tient essentiellement à la peur de les expédier dans le circuit de l'assistance en leur octroyant une allocation qui leur permettrait de ne pas travailler ou, tout du moins, réduirait leur motivation à le faire. La situation particulièrement délicate de certains jeunes à l'égard de la pauvreté n'est alors pas assurée par la solidarité publique.

Le refus d'attribuer l'assistance aux jeunes obéit donc à une logique de responsabilisation à l'égard de l'insertion sociale. Ils doivent faire leur preuve en mobilisant eux-mêmes les ressources pour leur bonne intégration sociale. Paradoxalement, l'exclusion des jeunes du droit au RMI les écarte de l'élargissement du socle des droits sociaux. "Il est frappant de constater que si le RMI a été voté dans un esprit de solidarité nationale et d'élargissement du socle des droits sociaux, il n'est toujours pas question en France de le concevoir comme un droit pour les jeunes" (Paugam, 2002a, p. XXVII). Le refus d'étendre le RMI aux plus jeunes creuse l'écart entre ceux qui disposent de nombreux atouts pour s'insérer, et ceux qui ne peuvent mobiliser les ressources nécessaires et qui se trouvent alors non seulement en

4. Parce que ce lien est vecteur de reconnaissance sociale (selon les théories de la reconnaissance d'Axel Honneth, 2000). Nous faisons encore plus référence aux travaux de Serge Paugam sur la place des différents types de lien social dans la reconnaissance sociale des individus et leurs modalités de participation à la société.

5. «En France, deux formes d'allongement des transitions vers l'âge adulte radicalement différentes et qui concernent des catégories de jeunes qui n’ont rien de commun, doivent être distinguées. La première peut être défnie comme une phase d'expérimentation. Elle concerne des jeunes qui ont poursuivi des études longues et qui se détachent progressivement de la dépendance familiale. [...] L'autre forme de prolongation se comprend beaucoup plus comme une forme de relégation. Elle concerne des jeunes et surtout des garçons qui ont arrêté leurs études le plus tôt et qui connaissent, à des degrés divers dans les pays d'Europe occidentale, une prolongation de l'adolescence» (Galland, 2000). 
situation d'échec à l'égard de l'insertion, mais aussi dans une situation économique souvent critique. Ainsi, l'exclusion des plus jeunes de ce droit cantonne les plus fragiles à la marge du marché de l'emploi, seul pourvoyeur de ressources économiques pour ceux qui ne peuvent bénéficier de la solidarité familiale.

En ne proposant que des aides ponctuelles, le système d'aide et d'accompagnement produit des ruptures dans les parcours de vie, en renvoyant les jeunes à leur autonomie ou au soutien de leurs parents ou de leurs proches, une fois les accompagnements terminés. Il produit de la discontinuité dans les parcours d'aide sociale, qui renforce le processus de ruptures cumulatives expérimentées par certains depuis l'enfance. De plus, le système d'aide aux jeunes est fragmenté à plusieurs égards. En effet, non seulement il est circonscrit dans le temps (la majorité des aides se trouve dans les dispositifs, par essence ponctuels, Ion \& Ravon 2005), mais il est aussi découpé en "secteurs d'aide " (santé, social, formation, emploi) dont la cohérence dépend de la qualité (inégale) des partenariats locaux (Loncle, 2011). Les travailleurs sociaux déplorent les ruptures institutionnelles que cette organisation produit pour les jeunes marginalisés et soulignent le manque de " passerelles " qui donneraient une cohérence globale à l'accompagnement, articulé autour de l'individu. Il leur est difficile de proposer un suivi linéaire et continu alors que le système d'aide est fractionné.

\subsection{Des dispositifs qui n'atteignent pas leur cible}

Les jeunes marginaux sont explicitement présentés comme le public cible de dispositifs comme le Fonds d'aide aux jeunes ou la Garantie jeunes. Un certain nombre de missions locales ont également mis en place des services d'accompagnement spécifiques "jeunes en errance ", actant d'une spécificité de ce public, à prendre en compte dans les accompagnements.

Pourtant, ils représentent également la part du public qui est mise en difficulté par les exigences des dispositifs, à la fois en raison de son éloignement des normes sociales qui façonnent l'intégration sur le marché du travail, mais aussi de la défiance qu'ils entretiennent à l'égard de ces normes, au fondement de leur mode de socialisation marginale. De fait, l'aide financière qui est apportée aux jeunes en difficulté est quasiment systématiquement associée à des mesures d'insertion professionnelle (Muniglia \& Rothé, 2012) ${ }^{6}$. Dans ce cadre, l'engagement individuel demandé constitue un frein pour les plus marginalisé : l'engagement contractuel, un travail en groupe de plusieurs mois et la durée même de l'engagement sont autant de conditions dissuasives pour des jeunes ancrés dans des sociabilités «zonardes» depuis de nombreuses années (Pimor, 2014).

6. Le RSA (Revenu de solidarité active) est ouvert aux jeunes actifs de moins de 25 ans qui ont travaillé au moins deux ans au cours des trois dernières années. La Garantie jeune, issue du Plan pluriannuel de lutte contre la pauvreté et pour l'inclusion sociale, de janvier 2013, semble poursuivre dans cette direction 
À cet égard, le fonctionnement de l'urgence sociale, dont les fondements sont notamment la permanence et l'inconditionnalité de l'accueil, met à distance les exigences normatives de l'insertion, et convient ainsi davantage aux jeunes socialisés dans la rue. La création et le maintien du lien social sont au centre de la relation d'aide, sans autre demande de contrepartie, la relation d'aide apparaît alors plus respectueuse du mode de vie des jeunes zonards (Rothé, 2016, 2018).

\section{L'infra-assistance adaptée aux jeunes marginaux}

L'infra-assistance, constituée notamment des dispositifs d'accueil inconditionnel de l'urgence sociale, est fréquentée régulièrement par les jeunes les plus marginalisés, alors qu'ils ne parviennent pas à "tenir " dans les dispositifs plus exigeants de seconde chance qui proposent une formation ou une aide dans l'accès à l'emploi. Ceci est lié à la tolérance des professionnels à l'égard des comportements des jeunes, acquis par la socialisation de rue, à savoir la vie au jour le jour qui rend impossible la prise de rendez-vous et la projection dans l'avenir, mais aussi le rejet des codes sociaux dominants qui régissent l'entrée dans l'âge adulte et définissent l'autonomie (celle-ci se traduisant par l'accès à un emploi, à un logement conventionnel et sédentaire, et plus largement par l'association emploi, autonomie financière et consommation de biens et de services).

Cependant, même pour les professionnels de l'urgence sociale, convaincus que l'insertion conventionnelle ne peut convenir à tous les jeunes, l'insertion reste un objectif de l'accompagnement qui se met en place au fil des rencontres. Le modèle de l'insertion via la formation, l'accès à l'emploi et au logement, est assoupli et remodelé au prisme de la marginalité ; son extrême normativité est mise en question. Pourtant, pour les intervenants, insérer ces jeunes dans la société, même de manière très marginale, constitue un horizon. Ils considèrent en effet que sans cet horizon, la rue est le seul avenir envisageable pour ces jeunes, et que cela n'est pas souhaitable. Dans ce sens, la fréquentation régulière des dispositifs de l'urgence sociale ouvre aux jeunes une porte vers la sortie de la zone, et par extension, vers l'acquisition d'une place sociale moins stigmatisée, voire plus reconnue.

\subsection{L'insertion en ligne de mire ?}

Les tensions croissantes qui s'insinuent dans l'exercice des métiers de l'intervention sociale (Chauvière, 2007) amènent les professionnels à questionner le sens des orientations qu'ils sont censés suivre. Ils interrogent notamment la pertinence de dispositifs, souvent expérimentaux, instables et inégalement dotés qui s'empilent au fil des changements politiques.

La forme même du dispositif interdit de penser la mise en place de l'action sur un temps long (Ion \& Ravon, 2005). De même, le caractère éphémère des outils à leur disposition 
ne semble pas adapté aux problématiques du public qu'ils accompagnent. Cette inadaptation suscite une réflexion sur l'imposition d'une norme qui semble pour certains inaccessible. Le discours politique présentant l'insertion comme un présupposé au bien-être des jeunes est notamment dénoncé par les professionnels comme un discours de «bonne conscience politique» :

"Oui, [nous sommes pris] dans un discours de normalité. A tout prix réintégrer des gens qui n'ont jamais été intégrés, c'est aussi paradoxal. Après on a plein de contradictions, plein de choses qui... Il suffit qu'on s'y arrête un petit peu et on se rend compte qu'on dit tout et n'importe quoi. Sous couvert politique, de la bonne conscience... Et puis cette normalisation, cette normalité. En plus, c'est une normalisation par le bas bien évidemment, éminemment par le bas. Avec: contentez-vous de ce qu'on vous donne et ne faîtes pas suer, c’est un peu ça. " (Infirmier, équipe mobile de psychiatrie précarité, la cinquantaine, expérimenté)

Dès lors, les injonctions à l'autonomie issues du discours social et politique à l'égard des jeunes en difficulté mettent les professionnels mal à l'aise avec une partie de leurs missions et des propositions qu'ils sont censés formuler auprès des jeunes accompagnés. Néanmoins, la jeunesse des usagers interdit de ne pas envisager leur avenir au travers d'une insertion sociale en dehors de la marginalité. Malgré leurs interrogations, les intervenants n'en poursuivent pas moins un double objectif, celui de permettre au jeune d'aller mieux, et s'adosser à ce mieux-être pour entreprendre des démarches d'insertion, les amenant à s'autonomiser de l'aide sociale. Être à l'écoute est donc également un enjeu pour le changement (Muniglia \& al. 2012). Pour les jeunes qui en auront les capacités, l'accompagnement deviendra plus responsabilisant, et la posture professionnelle se fera plus éducative.

\subsection{Le bas seuil, un format ad hoc}

Les professionnels travaillant auprès des jeunes désaffiliés mettent en avant la responsabilité du système dans l'émergence des situations d'exclusion quasi-totale de l'aide sociale. Ils considèrent que l'injonction à la normalisation par l'insertion, ainsi que la fragmentation des aides disponibles sont une source d'échec supplémentaire pour les jeunes les plus marginalisés. Pour endiguer ce processus, les intervenants des structures à bas seuil d'exigence profitent de la souplesse de leur fonctionnement pour adapter la proposition d'aide aux attentes et aux capacités des jeunes. Ils pensent alors la relation d'aide comme une possibilité de reconstruction d'un lien social, qui se définit en creux par rapport aux réponses institutionnelles et transcende leur fragmentation par une prise en charge de long terme.

Les structures dites "à bas seuil d'exigence " se caractérisent principalement par trois règles qui singularisent l'esprit de l'urgence sociale (Céfaï \& Gardella, op. cit.) : celle de l'inconditionnalité, selon laquelle toute personne en détresse ou en danger, quels que soient son statut et/ou son parcours, peut bénéficier d'une aide immédiate, sans contrepartie et sans projet d'insertion ; la règle de la durée limitée de l'hébergement d'urgence, qui vise à ne pas chroniciser l'accueil (mais qui produit un renforcement du cycle des ruptures) et la 
règle de la proximité des usagers, qui doit permettre de restaurer un lien social minimal. La règle de l'inconditionnalité et celle de la proximité sont traduites, en pratique, par l'instauration de la confiance dans la relation d'aide et la permanence de la présence des professionnels. Cette posture relationnelle est pensée comme un outil pour établir une relation de confiance qui pourra être la base d'une demande d'aide à l'insertion.

\subsubsection{L'inconditionnalité de l'accueil conditionne le maintien du lien social}

Les professionnels indiquent que la population des " jeunes en errance " éprouve beaucoup de difficultés à s'inscrire dans les trajectoires d'insertion conventionnelle proposées par les dispositifs, comme la mission locale, mais aussi dans les trajectoires plus adaptées proposées par ceux dit de "seconde chance ", comme la Garantie jeune, par exemple, qui peut être proposée à certains des jeunes marginalisés. Le travail de mobilisation ou de construction de compétences individuelles autour des " objets de resocialisation " que sont le travail, le logement, le retour dans la famille, ne fait pas sens pour ces jeunes trop éloignés des valeurs régissant leur mode de vie zonarde. Il ne fait pas davantage sens, du point de vue des professionnels, s'agissant de ce que ces jeunes peuvent atteindre comme objectif dans un premier temps. Comme beaucoup le répètent, "la marche à monter est trop haute " et voue toute tentative à l'échec.

Les dispositifs dits "à bas seuil d'exigence " prennent le contre-pied de ce mode de fonctionnement majoritaire dans les aides à l'attention des jeunes en difficulté d'insertion sociale et professionnelle. Grâce à leur caractère peu exigeant, les professionnels y travaillant peuvent se positionner davantage dans l'optique de reconstruire un minimum de relation sociale avec ces populations. Leur principe de fonctionnement valorise la relation d'aide sans contrepartie : "Il s'agit de sauvegarder un lien social avec des personnes en difficulté, quels que soient par ailleurs les comportements présents, passés ou à venir " (Naquet, 2000, p. 30). L'objet n'est pas, en premier lieu, l'insertion de l'individu, mais la « maintenance inconditionnelle du lien" avec lui.

Les professionnels expliquent mettre leurs pratiques au service de la relation immédiate qui se noue. Cette infirmière explique pourquoi le maintien du lien est nécessaire pour des jeunes qui, selon elle, n'ont pas grand-chose à quoi se raccrocher. Il s'agit en premier lieu de redonner une intégrité sociale à la personne, avant qu'elle puisse trouver la force d'entreprendre des démarches plus poussées et tournées vers l'avenir :

"Là, ils nont rien, ils nont rien et souvent la question c'est : pour qui ? Pourquoi? Ils n'ont pas de cadre de famille, très peu. Donc pour qui et pourquoi je ferais ça? C'est vrai que quand on n'a plus rien et qu'on a déjà rien et qu'on n'a plus de famille : pour qui ? Pourquoi? Pour aller mieux, oui. Mais combien de temps j'ai été mieux dans ma vie pour pouvoir dire que jai envie d'être comme ça?" (Infirmière, Centre d'hébergement et de réinsertion sociale - CHRS -, la cinquantaine, expérimentée). 
Les jeunes connaissant de graves difficultés depuis leur enfance sont vus par les intervenants comme ayant un besoin spécifique, celui de la création d'un lien social leur offrant une reconnaissance sociale permettant de se construire une identité et une utilité sociale, n'en ayant pas eu l'opportunité en raison d'une socialisation primaire plus confuse que fondatrice. Ce lien, ils le construisent dans la zone, au travers de la "Family ", le groupe de sociabilité des zonards décrit par Tristana Pimor (op. cit.), au travers d'une socialisation secondaire marginalisée. Pour les professionnels, tout l'enjeu est d'arriver à établir eux aussi un lien social vecteur de reconnaissance dans la relation d'aide qui ne soit pas assis sur ce qu'ils vont devenir, mais sur ce qu'ils sont aujourd'hui :

"On est dans ce contexte de sollicitude, en fait, parce qu'on ne leur demande pas grand-chose en échange, on leur demande juste de prendre soin d'eux. " (Psychologue, service d'aide aux femmes enceintes en difficulté, la quarantaine, expérimentée)

Cette façon d'envisager l'intervention prend le contrepied d'un redressement des conduites visant à faire intégrer aux individus les normes de leur bonne insertion. Il s'agit d'améliorer l'existence des personnes en situation de disqualification sociale (Paugam, 2002a) et d'aménager les conditions d'une meilleure cohabitation sociale. L'objectif est de leur faire une place dans la cité, sans pour autant leur demander de se conformer aux normes sociales dominantes (Vidal-Naquet, 2000).

\subsubsection{La confiance comme condition nécessaire de la prise en charge}

Par leur parcours, parce qu'ils vivent dans des circuits parallèles plus ou moins licites, qu'ils ont cumulé des déboires avec les services sociaux, la police et la justice, entamé plusieurs formations qui ont abouti à l'échec, ou encore parce qu'ils ont choisi de ne pas se conformer aux normes sociales, les jeunes marginaux nourrissent généralement une grande méfiance vis-à-vis des professionnels qui cherchent à s'approcher d'eux et, plus encore, à les aider. C'est pour cela que les professionnels considèrent l'instauration de la confiance comme un impératif préalable à l'établissement d'une relation d'aide durable :

"Je vais prendre cet exemple de cette jeune femme qui a vécu une enfance d'abandons répétés, qui n'arrive pas à faire confiance à beaucoup de personnes puisquelle a été trahie de nombreuses fois, donc il faut beaucoup de temps avant d'instaurer une confiance. Une fois que cette confiance elle est instaurée, on devient l'interlocuteur privilégié. Elle est hospitalisée actuellement, elle ne passe que par moi. " (Psychologue, équipe mobile de psychiatrie précarité, la cinquantaine, expérimenté).

Le fonctionnement des dispositifs d'urgence qui dispensent des ressources nécessaires au bien-être quotidien (repas, services d'hygiène, soins), favorise l'installation de la confiance grâce au contact régulier permis par la venue quotidienne des usagers. En effet, les pratiques des professionnels visent à "fidéliser » le public pour installer une relation de confiance, en mobilisant des techniques relationnelles basées sur la prise en compte des subjectivités individuelles. Il s'agit de discuter du quotidien, des évènements du jour, de prendre des 
nouvelles des uns et des autres. L'humour est très présent dans ces conversations usuelles et permet de maintenir un contact régulier en évitant d'aborder les raisons qui amènent les uns et les autres à demander de l'aide. Toutefois, au fil des échanges, la proximité qui s'installe autorise à parler des sujets plus sensibles, comme les projets d'avenir, le logement, la santé ou l'emploi. À cet égard, la confiance qui s'élabore au sein de la relation d'aide permet de "préparer les usagers aux épreuves publiques que leur réserve la société dans laquelle il convient de les (ré) insérer " (Breviglieri, 2005, pp. 225-226).

Effectivement, les usages que les jeunes font des structures et des aides ne perdurent souvent qu'au travers d'un lien de confiance qui se noue avec un professionnel. C'est ce lien qui les amène à revenir, et cela régulièrement, voir le même professionnel. Il constitue le principal outil mobilisé dans les pratiques d'accompagnement, où la contractualisation, l'inscription dans un projet ou encore la mise sous condition des aides ne sont pas de mise :

"Tout passe par là. Si on n'est pas en mesure de proposer une relation de confiance, un minimum, une relation humaine, chaleureuse, ça ne sert à rien, voilà : un minimum. Essayez de créer un contact, une relation exploitable pour présenter ce qu'on peut offrir en termes de services. Moi, je propose du soin pour les toxicomanes, à moi de construire la relation qui me permette d'établir ce soin-là. Tout repose sur la relation, la qualité, comment est-ce quion est en mesure, capable, d'établir des relations suffisamment costauds pour proposer un parcours qui est une épreuve, c'est sûr. » (Infirmier de liaison en toxicomanie, la quarantaine, expérimenté)

Dans ce sens, les professionnels s'emploient à réunir les conditions pour tisser un lien minimum suffisamment solide pour établir une relation qui fidélise le public. C'est sur la base de ce lien qu'ils construisent un accompagnement qui rassure le jeune usager sur le fait qu'il ne devra pas s'engager au-delà de la relation d'aide elle-même...

\subsubsection{La constance des professionnels pour garder le lien}

La permanence de la présence des professionnels est une autre dimension, à la fois complémentaire et indispensable à la confiance, qui est considérée comme un garant fort du lien qui s'établit avec les usagers. Du point de vue des intervenants sociaux, garder la porte ouverte, c'est pallier la logique éphémère des dispositifs d'aide à l'insertion.

Cependant, cette permanence du lien n'est envisageable que si le professionnel reste des années au même poste et au même endroit. Il devient une personne de référence qui sera sollicitée au titre du lien qui a été construit et non pas seulement au titre de sa position professionnelle. Alors, la constance des professionnels et la confiance que les usagers leur accordent sont posées comme les ingrédients indispensables à la " fidélisation ", qui rendra des démarches d'insertion envisageables. Cette posture spécifique adoptée par les professionnels - un accueil inconditionnel, un accompagnement adapté aux subjectivités personnelles - favorise de fait un suivi de long court, même s'il est fragmenté. 
Beaucoup de professionnels travaillant selon cette logique nous font part de cas de personnes accompagnées revenant les voir des années plus tard, et ce à plusieurs reprises, alors que rien ne les y obligeait, si ce n'est l'historique de leurs relations :

"Ils ont cet espèce de réflexe de revenir ici, je ne sais pas pourquoi. Ça m'a toujours interrogée ça. Et, ils débarquent, neuffois sur dix, comme ça dans le hall, et on les a des fois pas vus pendant cinq ans ou dix ans et ça m'ahurit toujours parce que, statistiquement, je ne devrais plus être là, car les assistantes sociales dans un service d'urgence qui restent autant de temps que moi... et je trouve ça très comique que pas une seconde, ils se disent que je puisse être claquée, mutée... Et qu'il n'ait même pas l'idée d'appeler. Ça m’interroge à chaque fois. " (Assistante sociale, Permanence d'accès aux soins de santé - PASS - , la cinquantaine, expérimentée).

Le jeune ne sollicite pas une aide concrète proposée par un dispositif spécifique, mais une personne qu'il connaît. Cette posture consolide le rôle de " tampon " joué par les travailleurs sociaux qui adoptent ces principes d'intervention. Là où les dispositifs d'aide à la formation ou à l'insertion professionnelle accompagnent sur une courte durée, donnent des compétences à l'individu, puis lui laissent la charge de poursuivre seul ses démarches une fois l'accompagnement terminé, les professionnels positionnent les structures à «bas seuil d'exigence " comme un relais pérenne, une passerelle faisant la jonction entre les dispositifs durant les périodes de décrochage. La constance des professionnels est ainsi érigée en véritable outil de maintien du lien social, de lutte contre la désaffiliation et d'accompagnement social.

\section{Adapter I'insertion}

Nous venons de le voir, les professionnels de l'urgence mobilisent les principes de fonctionnement $\mathrm{du}$ "bas seuil d'exigence " afin d'accompagner les jeunes, alors que ces dispositifs sans contrepartie n'imposent pas l'obligation de venir régulièrement. Forts de ce constat, nous nous intéressons plus spécifiquement aux représentations motrices de la volonté de mettre en place un accompagnement social pour les jeunes marginalisés. L'analyse des entretiens professionnels éclaire la place prise par l'objectif d'insertion dans les pratiques auprès des jeunes.

Dès lors, au sein des dispositifs de l'urgence sociale, des spécialisations en direction de la jeunesse apparaissent via l'orientation qui est donnée à l'accueil des plus jeunes, pour lesquels l'installation dans l'assistance semble à éviter absolument. Alors que les « exclus » plus âgés bénéficient d'une prise en charge au jour le jour, dont l'objectif est de réduire au maximum les dommages liés au processus d'exclusion sociale (même si leur sont proposées des mesures de réadaptation sociale), les plus jeunes font l'objet d'une prise en charge davantage tournée vers la mise en place de la possibilité, pour eux, de s'insérer. De plus, l'importance de la population jeune, au sein du système de l'infra assistance, conduit 
à une recomposition des dispositifs autour de mesures spécifiquement dédiées aux plus jeunes. L'individualisation des accompagnements y favorise la mise en place de démarches aux exigences abaissées, sensibilisant les jeunes marginalisés aux comportements propices à l'accès à une formation ou à un emploi (se lever le matin, soigner ses addictions, être soucieux de son hygiène, par exemple).

L'accès à une formation ou un emploi restent des horizons, même lointains, pour les jeunes marginalisés. Ainsi, la transmission d'un ensemble de compétences et de comportements que les jeunes n'ont pas acquis au cours de leur socialisation devient un objectif en creux de l'accompagnement, sans que celui-ci soit pour autant tourné directement vers l'accès à la qualification ou à l'emploi. L'objectif des professionnels est de rendre plus aisée leur éventuelle intégration aux dispositifs de préparation à la qualification (comme la Garantie jeune) et de lever les freins majeurs à l'emploi que peuvent représenter le fait de dormir dehors, l'alcoolisation massive quotidienne, la possession de chiens, ou encore une hygiène mal adaptée à la vie collective d'une classe ou d'un bureau. L'analyse des pratiques professionnelles révèle que le lien qui se noue dans la relation d'aide est le principal levier qu'activent les intervenants sociaux lorsqu'ils proposent des pistes pour faire évoluer les comportements qu'ils jugent les plus délétères pour l'avenir des jeunes.

\subsection{Réduire les risques liés à la marginalité}

Pour les professionnels, la confiance établie est une opportunité pour envisager des démarches liées à l'insertion. Celles-ci, adaptées à chacun, visent à ne pas majorer les difficultés des usagers. De leur point de vue, abaisser les contraintes est une manière d'introduire de l'équité dans les accompagnements, là où les politiques de l'insertion se fondent sur le principe d'égalité qui ne permet pas de prendre en compte la variété des expériences de vie.

Pour cela, les intervenants jugent nécessaire de redéfinir la norme de l'insertion au regard de parcours de vie des jeunes usagers, marqués par des ruptures en chaînes, notamment avec les institutions sociales. Plutôt que de travailler la dimension du projet de vie ou de formation, ou encore de lever les freins à une entrée en emploi ou en formation, ils cherchent davantage à réduire les risques liés à un mode de vie marginal qu’à insérer les jeunes en les resocialisant aux codes d'une vie plus conventionnelle. Le plus difficile étant de réussir à rencontrer régulièrement un public très fuyant, les professionnels pensent les aides dispensées dans les accueils de jour comme des produits d'appel, qui sont en fait des supports à l'installation d'une relation. Dans la plupart des structures d'urgence, le ciblage n'est pas fait par population, mais par le type d'aide proposée : hygiène, toxicomanie, restauration, orientation, logement... Dans ce cadre, les professionnels font de l'aide concrète une passerelle vers une prise en charge plus globale et plus efficace. Ils l'envisagent comme un « outil d'appel » qui prend la forme d'un prétexte pour établir une relation plus durable. Beaucoup d'intervenants présentent leur action de cette manière. 
"Et on se sert un petit peu de cette aide alimentaire comme outil d'appel, on va dire, pour travailler sur l'ébauche des parcours d'insertion des personnes. " (Responsable restaurant social municipal)

Un chargé de projet dans un accueil de jour utilise quasiment la même expression :

"Le lieu d'accueil de jour est un lieu basé uniquement sur l'hygiène, le produit d'appel, c'est l'hygiène, c'est des douches, des machines à laver, des sèches linges, des consignes, et puis, un petit lieu qui se dit un peu convivial... et puis... voilà, on dit souvent que c'est un piège à con, pourquoi ? Parce qu'on n'a pas mis des lavandières du Portugal à tenir ce truc-là! On a mis des éduc, des assistants sociaux et des infirmiers. " (Chargé de projet, accueil de jour)

Les aides matérielles sont considérées comme un support à la discussion. De ce fait, les structures de l'infra-assistance permettent un accès au droit facilité, en assouplissant le fonctionnement des institutions, notamment grâce à la valorisation de l'écoute, de l'empathie et des subjectivités individuelles. Le seuil d'exigence requis pour y être reçu est beaucoup moins élevé que pour intégrer des dispositifs appliquant une logique bureaucratique où les règles et contraintes d'accès sont les mêmes pour tous. Les règles y sont également plus souples que dans les dispositifs où elles sont déjà assouplies, mais requièrent tout de même des compétences, comme la ponctualité ou la capacité à être en groupe. Une assistante sociale qui accompagne des femmes enceintes en difficulté explique comment elle assouplit les règles pour celles qu'elle juge les plus fragiles :

"On ne donne pas de rendez-vous à neuf heures du matin, on essaie de les mettre en début d'après-midi, parce que les rendez-vous loupés : elle ne s'est pas levée... il y a aussi le côté adaptabilité mutuelle. C'est-à-dire on fait en sorte de leur rendre les choses possibles aussi, ça fait partie de notre façon de bosser [...] on leur donne les moyens progressivement de se saisir des outils d'insertion. Alors ça passe par le soin, les horaires petit à petit, parce que ça les prépare progressivement à avoir des horaires un petit peu plus réguliers. " (Assistante sociale, service d'aide aux femmes enceintes en difficulté, la quarantaine, expérimentée)

Ainsi, par la prise en compte des singularités individuelles, les professionnels créent une marche supplémentaire vers le droit commun, qui tient compte des possibilités individuelles. Le "bas seuil d'exigence " est pour eux un support qui permet de faire prévaloir le principe d'équité, pour atteindre l'égalité dans l'accès aux droits. C'est en ces termes que l'intervention est pensée :

"Donc, il y avait quand même le souhait de leur permettre de se réinsérer sans mettre la barre trop haut pour que ce soit jouable pour tout le monde. " (assistante sociale, service d'aide aux femmes enceintes en difficulté, la quarantaine, expérimentée)

Prenant le contre-pied d'une injonction trop forte à la normativité, le fonctionnement des structures de l'infra-assistance répond au principe de réduction des risques afin de permettre aux usagers d'accéder à un "mieux-être " (plutôt qu'un bien-être). Par la valorisation d'une posture empathique, les intervenants cherchent à atténuer la vio- 
lence de l'imposition de l'impératif moral de l'insertion, en le rendant plus accessible grâce à la création d'un lien social suffisamment structurant. Ce positionnement tactique d'adaptation à la subjectivité des jeunes fait des dispositifs de l'urgence des lieux d'accès aux droits (Chauveaud \& al., 2011) abaissant les seuils d'exigence de l'insertion pour les rendre plus accessibles.

\subsection{Réguler les démarches d'insertion}

Dans les cas où les intervenants considèrent que les demandes d'insertion par la formation et l'emploi se font trop pressantes, ils endossent un rôle de "tampon " pour adoucir les démarches jugées irréalistes. Selon eux, certains jeunes sont engagés trop précocement dans des formations, ou trouvent un emploi via la mission locale par exemple, ce qui les met souvent en situation d'échec quand leurs autres problèmes n'ont pas été pris en charge (souffrance psychologique, toxicomanie, logement stable, etc). De manière générale, les professionnels rencontrés dans le secteur de l'urgence sociale considèrent que la priorité est davantage de "prendre soin de soi ". Ils l'envisagent comme un préalable à l'engagement dans des démarches de formation ou de recherche d'emploi.

Dès lors, ils jouent le rôle de régulateur de ces demandes en les adaptant à la situation de la personne accompagnée. Très souvent, des démarches d'insertion déjà engagées sont mises en attente afin de traiter efficacement un problème plus profond, affectant directement l'insertion. Un infirmier en conduites addictives pointe le conflit qui peut s'installer entre les logiques de l'insertion et les logiques du soin, par défaut de concertation entre les acteurs. Il évoque l'exemple d'une jeune femme qu'il suit depuis longtemps pour ses problèmes de toxicomanie. Elle est à la rue depuis plusieurs années et vient tout juste d'obtenir un logement. De ce fait, la mission locale a pu lui trouver une formation pour laquelle elle doit se rendre en stage. Cependant, le stress généré par cette nouvelle formation, ainsi que l'entrée en cure de soin en toxicomanie très récente (elle est traitée par substitution) la rendent très fragile, à tel point qu' elle a recommencé à consommer toutes sortes de drogues. L'infirmier est alors intervenu pour interrompre la démarche de formation en faisant du soin une priorité. Selon lui, sans arrêt de la toxicomanie et stabilisation de l'état psychologique de la jeune femme, aucune démarche de formation n'est viable :

"Voilà, oui, c’est ce qui s'est passé avec $N$ : passage sous méthadone, arrêter de picoler... Je leur ai dit 'arrêtez les stages, stop'... Mais ça, ils comprennent très bien que quand mes affaires marchent, les leurs, elles ne marchent pas, c'est sûr." (Infirmier de liaison en toxicomanie, la quarantaine, expérimenté)

Pour ces professionnels du bas seuil, la dimension très prégnante du " projet » dans les dispositifs d'aide aux jeunes représente également un frein dans l'accès aux démarches pour des jeunes en souffrance psychologique. Il s'agit alors d'adapter cette demande 
de projet, en la décentrant de l'objectif de la formation ou de la recherche d'emploi, en l'élaborant davantage comme un instrument de construction de la confiance en soi. Les projets d'acheter un camion pour se déplacer de festival en festival ou de se projeter dans une carrière d'artiste sont alors discutés comme tels. Adapté aux réalités de la vie et des envies du jeune reçu, le projet n'est pas d'emblée jugé irréaliste. Support de la contractualisation, il est adouci et singularisé et accueille les idées les plus éloignées de la norme d'insertion, sans les juger. Les intervenants le mettent au service de l'émergence de l'envie de se projeter dans un avenir, proche ou non. Voici ce que nous dit une assistante sociale au sujet des jeunes à la rue qu'elle reçoit. Elle souligne la difficulté, pour la plupart, d'élaborer le projet d'insertion demandé pour accéder à un hébergement, alors qu'il leur faut d'abord trouver des conditions de vie acceptables pour pouvoir se projeter vers l'avenir :

"C'est vrai que la difficulté elle est là, notamment pour tous ces jeunes, que l'on appelle les incasables. C'est compliqué parce que tout établissement, toute structure, va demander : c'est quoi son projet? Mais tout le problème, il est là. C'est qu'il faut le définir avec lui, ce que serait son projet." (Assistante sociale de secteur, la cinquantaine, expérimentée)

\subsection{Préparer malgré tout aux exigences de l'insertion professionnelle}

L'usage assoupli du contrat d'insertion est un bon exemple de stratégies professionnelles mises en place au sein des dispositifs de l'infra-assistance. Le bricolage consiste ici à contourner le caractère excluant de la contractualisation pour des jeunes ne se projetant pas dans l'avenir. Le détournement de la rigidité de la logique contractuelle repose sur une adaptation des clauses du contrat aux besoins du jeune usager. Ceux-ci sont définis à la faveur de la relation d'aide et de l'introduction de la subjectivité dans la définition des termes de l'engagement.

Le contrat se fait « incluant » et devient un outil progressif d'adaptation aux contraintes du dispositif. Plus largement, il devient un outil au service d'une éducation des conduites, qui vise à donner aux jeunes une voie d'accès aux compétences nécessaires à une insertion sociale en dehors du monde de la zone : "Le coût engendré pour répondre aux exigences capacitaires du contrat est en effet rendu acceptable dans la mesure où s'annonce un sens positif donné à une vie reconnue comme choisie, révisée et justifiée. Le travailleur social demeure en cela l'essentiel premier témoin de l'individualisation du parcours d'insertion, la source d'un faisceau majeur de reconnaissance " (Breviglieri, op. cit., p. 229).

Dans les centres d'hébergement et de réinsertion sociale (CHRS), la recherche active d'une formation ou d'un emploi est un des engagements à prendre pour être hébergé temporairement. Cette exigence met en échec de nombreux jeunes qui se débattent davantage avec leur toxicomanie ou les histoires du passé qu'avec le choix d'une voie professionnelle. Dès lors, la contractualisation se fait sur l'engagement dans une 
démarche de projet de vie et sur des petits objectifs, plus que sur des résultats tels que l'entrée en formation ou l'obtention d'un contrat de travail. Les entretiens préalables à la rédaction commune du contrat visent justement à faire émerger ce projet et à évaluer les capacités d'action des jeunes accueillis. Il s'agit de ne pas les mettre en échec par un engagement qu'ils ne pourraient pas honorer. Pour cette éducatrice d'un CHRS, il s'agit d'offrir au jeune la possibilité d'expérimenter l'autonomie avec un filet de sécurité :

"Et ça, je pense que c'est important, surtout avec les 18-20 ans, [...], mais je pense que notre rôle en tant que professionnel de l'accompagnement des jeunes adultes, c'est aussi leur permettre, dans cette période où ils sont un peu entre adulte, ado, de leur dire : 'maintenant tu es adulte, tu dois assumer des responsabilités, ici tu es dans un espace où tu peux les expérimenter parce que tu as un filet finalement, tu as des guides, des filets, mais à toi de les expérimenter aussi. Voilà, plus dans une position un petit peu bienveillante " (Éducatrice CHRS, la vingtaine, début de carrière).

Le contrat se structure autour d'une réadaptation des comportements aux exigences du monde du travail. C'est l'employabilité des jeunes qui est travaillée. La restauration de la confiance en soi est aussi celle d'un certain nombre de compétences sociales attendues de jeunes adultes aujourd'hui.

\section{Conclusion}

Le principe d'inconditionnalité des dispositifs à bas seuil d'exigence révèle, par contraste, que la deuxième chance est conditionnée, dès le départ, à la capacité des jeunes à mobiliser des qualités et des compétences qui seront le support des trajectoires d'insertion. Si ces capacités font défaut, l'action des dispositifs de deuxième chance sera inefficace, voire contreproductive, en induisant une rupture sociale supplémentaire dans des parcours déjà heurtés.

La Garantie jeune reçoit notamment un certain nombre de jeunes marginaux ayant vécu dans la "zone " durant de nombreuses années. Certains d'entre eux sont mis en difficulté par la formalisation d'un projet professionnel qui sera jugé réaliste, ou encore par la nécessité de travailler en groupe. La durée de l'accompagnement formalise également une difficulté supplémentaire au moment de son arrêt. En effet, l'absence d'accompagnement, par la suite, amène une part des jeunes à retrouver les situations de vulnérabilité, voire de marginalité, qu’ils ont connues auparavant.

Ainsi, alors que les orientations de l'action publique visent à consolider les parcours fragiles, les mesures en direction des jeunes expérimentant des ruptures récurrentes déstabilisent davantage les trajectoires qu'elles ne les réassurent.

Comme ils ne mobilisent pas les ressources demandées par les mesures sociales d'accompagnement - par défiance ou par manque de ressources - ils basculent rapidement 
vers un recours routinier à l'infra-assistance. Ainsi, les jeunes marginaux ne sont pas touchés par les dispositifs de seconde chance, notamment parce que le cumul des difficultés qu'ils expérimentent depuis l'enfance et une socialisation de rue les amènent à valoriser un mode de vie alternatif où l'école et le travail ne sont pas des valeurs centrales.

De ce fait, les accompagnements socio-éducatifs visant à remobiliser les jeunes pour qu'ils obtiennent une qualification touchent très peu ce public. Cependant, s'ils ne sont pas pris en charge au moment où ils "décrochent " de l'école, leur fréquentation de la "zone " les amène vers un type d'accompagnement plus informel qui va se mettre en place dans la relation qui s'établit avec les intervenants de l'urgence sociale. L'attention de ces derniers se porte sur le maintien du lien social et la reconstruction de soi au travers d'une relation humaine sans engagement. Leur posture professionnelle, attachée au maintien d'un lien social valorisant pour les jeunes qu'ils rencontrent, et les pratiques de "fidélisation " des usagers, assises sur une posture d'écoute compréhensive, constituent en elles-mêmes un accompagnement. C'est ainsi que, au travers de la valorisation de la relation d'aide plus que de l'aide concrète, les professionnels tentent de résoudre le paradoxe de l'injonction à l'autonomie et à l'insertion qui tient à l'écart les jeunes vulnérables. Ils se font les artisans d'une régulation du droit "par le bas" (Warin, 2002), visant à assouplir les normes en les adaptant aux contraintes réelles des jeunes qu'ils rencontrent (socialisation marginalisée, grande fragilité psychologique, conditions de la vie à la rue). Ils fabriquent ainsi la possibilité d'une seconde chance, sans adosser leur intervention aux principes forts de l'acquisition de l'autonomie financière et résidentielle, du projet ou de la responsabilisation qui règnent aujourd'hui.

Si les professionnels sont attachés avant tout à leur mission sociale, la jeunesse du public les amène à bricoler des solutions qui s'apparentent à un accompagnement vers l'insertion. Ils vont travailler à faire évoluer les comportements des jeunes de manière douce et adaptée, afin de ne pas heurter les biographies personnelles, et ce, sans contrepartie - l'aide n'étant jamais conditionnée à l'évolution des comportements ou à l'acquisition de compétences spécifiques.

Un paradoxe naît cependant de cet accompagnement singulier car le lien qui est noué au sein de la relation d'aide est voué à être défait au moment de l'autonomisation des jeunes gens dans leurs démarches. Si la fin de l'accompagnement signe sa réussite pour l'action publique, il n'a définitivement pas le même sens pour les jeunes usagers. La fin de l'accompagnement est vécue par beaucoup comme une rupture supplémentaire qu'ils cherchent à éviter en ne quittant pas les dispositifs d'urgence.

Cet attachement au lien plus qu'aux démarches d'insertion qu'il permet explique l'enfermement de certains jeunes dans le circuit de l'infra-assistance. Prisonniers de cette inscription chronique dans le recours à l'infra-assistance, ils s'installent dans une carrière de marginalité et s'éloignent de l'objectif du retour vers le droit commun. 


\section{- Bibliographie}

Breviglieri M. (2005), «Bienfaits et méfaits de la proximité dans le travail social », in Ion J. (dir.), Le travail social en débat[s], Paris, La Découverte, pp. 219-234.

Céfaï D., Gardella E. (2011), Lurgence sociale en action, ethnographie du samu social de Paris, Paris, La Découverte.

Chauveaud C., Mazet P., Warin P. (2011), « Des fabriques d'accès au(x) droit(s) ", La Lettre de l'ONPES, n ${ }^{\circ} 3$, avril.

Chauvière, M. (2007), Trop de gestion tue le social. Essai sur une discrète chalandisation, Paris, La Découverte.

Denecheau, B, Houdeville G., Mazaud C (2015), A l'école de l'autonomie, Épreuves et enjeux des dispositifs de deuxième chance, Paris, L'Harmattan.

Dubar C. (2001), « La construction sociale de l'insertion professionnelle en France ", in Roulleau-Berger L., Gauthier M., (dir.), Les jeunes et l'emploi dans les villes d'Europe et d'Amérique du nord, Paris, Ed. de l'aube, pp. 111-123.

Galland O. \& Cellule de sociologie de l'OFCE (2000), « Une polarisation de la jeunesse française ", in Revue de l'OFCE, $\mathrm{n}^{\circ}$ 72. pp. 221-228.

Honneth A. (2000 [1992]), la Lutte pour la reconnaissance, Paris, Cerf.

Ion J., Ravon B. (2005), "Institutions et dispositifs », in Ion J. (dir.), Le travail social en débat $[s]$, Paris, La Découverte, pp. 71-86.

Lima L. (2012), "Les jeunes vulnérables, laboratoire de l'Etat social actif ? ", in INJEP, Rapport de l'Observatoire de la jeunesse, Paris, La Documentation française, pp. 186-200.

Lima L. (2008), « Le temps de la prime insertion professionnelle : un nouvel âge de la vie " ?, in Guillemard A.-M. (dir.), Où va la protection sociale?, Paris, Presses universitaires de France, pp. 49-67.

Lima L. (2004), L'Etat social et les jeunes : une comparaison France-Québec des systèmes d'assistance-jeunesse, thèse en sociologie, Université Aix-Marseille II de la Méditerranée.

Loncle P. (2011), "La jeunesse au local. Sociologie des systèmes locaux d'action publique ", Sociologie, 2, pp. 129-147.

Muniglia V., Rothé C. (2012), "Jeunes vulnérables : quels usages des dispositifs d'aide ", Agora Débats/Jeunesses, Paris, Presses de SciencesPo. / INJEP, nº 62, pp. 65-79. 
Muniglia V., Rothé C. (2013), " Parcours de marginalisation de jeunes en rupture chronique : l'importance des autrui significatifs dans le recours à l'aide sociale ", Revue Française des Affaires Sociales, n 1-2, pp. 76-95.

Muniglia V., Rothé C., Thalineau A. (2012), «Accompagner les jeunes vulnérables : catégorisation institutionnelle et pratique de la relation d'aide ", Agora Débats/jeunesses, Paris, Presses de Sciences Po./INJEP, nº 62, pp. 97-110.

Muniglia V. (2015), Devenir adulte quand le soutien familial fait défaut. Sociologie d'une jeunesse vulnérable, thèse en sociologie, Paris, EHESS.

Paperman P., Laugier S. (dir.) (2011), Le souci des autres. Éthique et politique du care, nouvelle édition augmentée, Paris, EHESS.

Parazelli M. (2002), La rue attractive. Parcours et pratiques identitaire des jeunes de la rue, Sainte-Foy, PUQ.

Pattegay P. (2001), "L'actuelle construction, en France, du problème des jeunes en errance. Analyse critique d'une catégorie d'action publique ", Déviance et Société, Vol. 25, n³ pp. 257-277.

Paugam S. (2002a) [1991]), La disqualification sociale, Paris, PUF.

Paugam S. (2002b), La société française et ses pauvres, Paris, PUF.

Paugam S. (2008), Le lien social, Paris, PUF.

Pimor T. (2014), Zonards, une famille de rue, Paris, Presses universitaires de France.

Rothé C. (2016), Jeunes en errance. Relation d'aide et carrières de marginalité, Rennes, Presses Universitaires de Rennes.

Warin P. (2002), «Les dépanneurs de justice. Les ' petits fonctionnaires' entre qualité et équité ", revue Droit et Société, vol. 33.

Vidal-Naquet P.-A. (avec la coll. de Laval C.) (2000), Sur les chemins de l'errance estivale, ministère de l'Emploi et de la Solidarité, diffusion DGAS. 